\title{
Correlation between emission intensity of self-assembled germanium islands and quality factor of silicon photonic crystal nanocavities
}

\author{
N. Hauke, ${ }^{1, *}$ S. Lichtmannecker, ${ }^{1}$ T. Zabel, ${ }^{1}$ F. P. Laussy, ${ }^{1}$ A. Laucht,${ }^{1}$ M. Kaniber, ${ }^{1}$ D. Bougeard,${ }^{2}$ G. Abstreiter,,${ }^{1,3}$ \\ J. J. Finley, ${ }^{1}$ and Y. Arakawa ${ }^{3,4}$ \\ ${ }^{1}$ Walter Schottky Institut and Physik Department, Technische Universität München, Am Coulombwall 4, D-85748 Garching, Germany \\ ${ }^{2}$ Institut für Experimentelle und Angewandte Physik, Universität Regensburg, D-93040 Regensburg, Germany \\ ${ }^{3}$ Institute for Advanced Study, Technische Universität München, D-85748 Garching, Germany \\ ${ }^{4}$ Institute of Industrial Science, Institute for Nano Quantum Information Electronics, The University of Tokyo, 4-6-1 Komaba, \\ Meguro-ku, Tokyo 153-8505, Japan
}

(Received 19 March 2011; revised manuscript received 19 May 2011; published 29 August 2011)

\begin{abstract}
We present a comparative microphotoluminescence study of the emission intensity of self-assembled germanium islands coupled to the resonator mode of two-dimensional silicon photonic crystal defect nanocavities. The cavity-mode intensity is investigated for L3 and hexapole cavities with a range of different mode quality factors. For each type of cavity, many nominally identical samples are probed to obtain reliable statistics. As the cavity-mode quality factor increases, we observe a clear reduction of the average mode emission intensity under conditions of strong optical pumping. This clear trend is compared with simulations based on a dissipative master-equation approach that describes a cavity weakly coupled to an ensemble of emitters. We obtain direct evidence that reabsorption of photons in the cavity is responsible for the observed trend. When combined with the observation of cavity linewidth broadening in power-dependent measurements, we conclude that free carrier absorption limits the cavity-mediated light enhancement under conditions of strong excitation.
\end{abstract}

DOI: 10.1103/PhysRevB.84.085320

PACS number(s): 42.60.Da, 42.70.Qs, 78.55.-m, 42.50.Ct

\section{INTRODUCTION}

The development of an efficient silicon- (Si-) based light source is of great interest since it would pave the way toward the development of optical interconnects that are compatible with standard CMOS technologies. ${ }^{1-5}$ Due to its indirect band gap, interband recombination in crystalline Si requires the participation of phonons to conserve crystal momentum. This leads to a very low internal quantum efficiency and $\mathrm{Si}$ is, therefore, rarely used as active light-emitting material. ${ }^{6}$ One approach that has been explored is to enhance the material radiative emission efficiency by exploiting cavity quantum electrodynamics effects using photonic crystal $(\mathrm{PhC})$ nanocavities. Enhanced photoluminescence (PL) has been recently reported in crystalline $\mathrm{Si} \mathrm{PhCs}$ (Refs. 7-10) as well as for germanium (Ge) islands embedded in a $\mathrm{Si} \mathrm{PhC}$ nanocavity. ${ }^{11-15}$ However, detailed investigations of the nature of the light-matter coupling in such systems have not been performed in either theory or experiment. For example, comparatively little is known about the emissive properties of Ge islands in nanocavities due to a lack of information about their natural emission linewidth or effective oscillator strength. The use of $\mathrm{PhC}$ nanostructures may provide a route to enhance optical activity to a level where optical properties of single Ge islands can be directly investigated.

In this paper, we report on the investigation of the cavityenhanced emission from self-assembled Ge islands grown by molecular beam epitaxy (MBE) in PhC nanocavities. We begin by comparing the emission intensities in PL experiments from L3 and hexapole PhC cavity modes under comparable conditions of optical pumping as a function of their mode quality $(Q)$ factors. Averaged over a large number of different but nominally comparable cavities, a general finding of our work is that an increase of the average mode PL intensity is observed as the mode $Q$ factor becomes smaller under conditions of strong optical pumping. Using weaker pump powers, this trend becomes less pronounced. In order to understand this very clear experimental observation, we introduce a model based on a dissipative master equation for an ensemble of emitters, where each emitter is in a highly excited state to account for the strong optical pumping and weakly coupled to the cavity mode to account for the expected low oscillator strength of the Ge islands. The model exhibits various regimes with distinct dependencies between mode intensity and $Q$ factor, depending on the spectral emitter ensemble distribution and the emitter-cavity detuning. By comparing this model with the experimentally observed trend, we obtain evidence that reabsorption of photons in the cavity is responsible for the observed $Q$ factor dependence of the intensity. Precisely this effect ultimately limits the radiative efficiency in such systems. By fitting the predictions of our model to our data, we extract a photon reabsorption time of $\tau_{\text {abs }}=2.4 \pm 0.6 \mathrm{ps}$ for an optical excitation power density of $600 \mathrm{~kW} / \mathrm{cm}^{2}$, in very good agreement with the photon reabsorption time $\tau_{\mathrm{abs}}^{\star}=$ $2.9 \pm 0.6 \mathrm{ps}$ extracted from power-dependent measurements of the cavity-mode linewidth. This suggests that free carrier absorption (FCA) takes place under the conditions of strong optical pumping in the cavities. Hence, we show that cavity modes with high $Q$ factors and, thus, long photon lifetimes exhibit a reduced radiative efficiency under conditions of strong pumping as compared to cavity modes with lower $Q$ factors.

\section{SAMPLE STRUCTURE AND EXPERIMENT}

The sample consists of a two-dimensional (2D) $\mathrm{PhC}$ that is fabricated into a freely suspended $\mathrm{Si}$ slab, which contains a single layer of self-assembled MBE grown Ge islands as illustrated in Fig. 1(a). The fabrication starts from a 
(a)

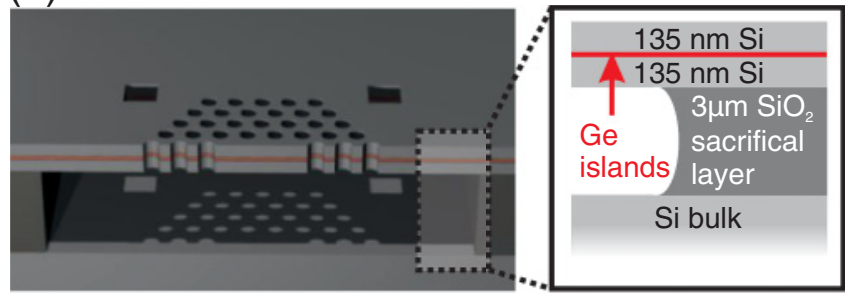

(b)
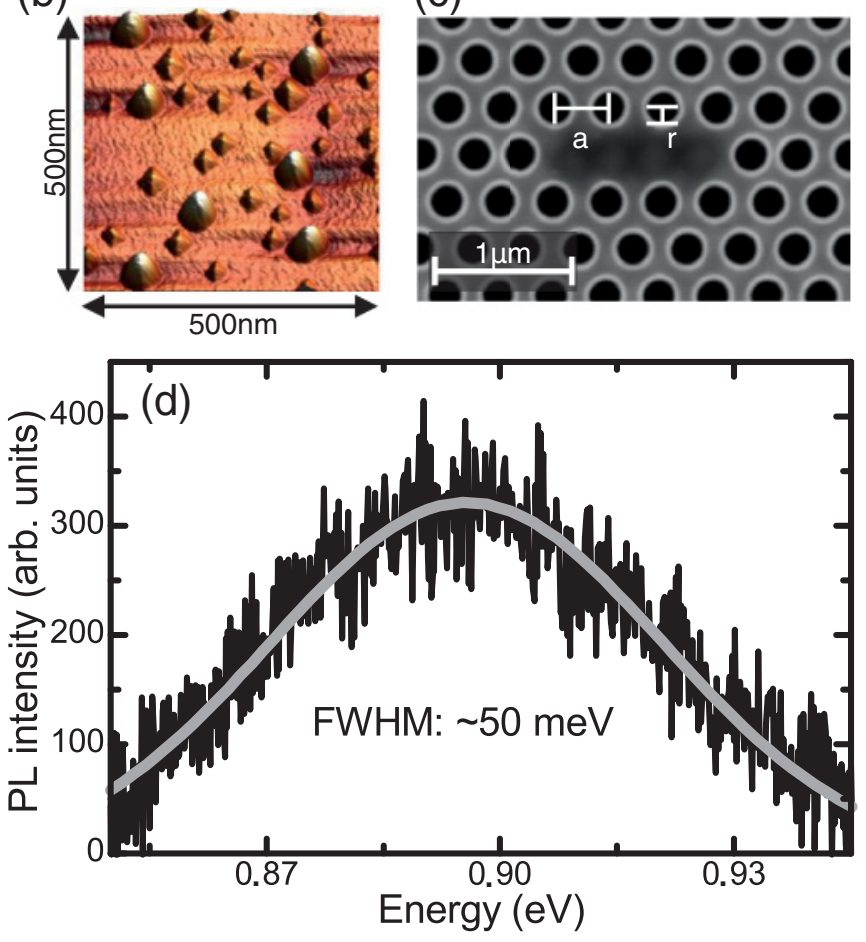

FIG. 1. (Color online) (a) Left panel: Schematic cross-sectional representation of the photonic crystal nanocavity structures investigated. Right panel: layer sequence in the active region. (b) Atomic force microscope image of the Ge islands investigated. (c) SEM image showing a L3 photonic crystal cavity with shifted outer holes from the top. (d) $\mu \mathrm{PL}$ spectrum from Ge islands in the unpatterned region at $T=25 \mathrm{~K}$. The grey line is a Gaussian fit to the data.

silicon-on-insulator (SOI) wafer provided by Soitec $^{16}$ with a 220 -nm-thick crystalline Si layer on top of a 3- $\mu$ m-thick layer of buried $\mathrm{SiO}_{2}$. Before growth, the crystalline silicon layer is thinned to $50 \mathrm{~nm}$ using isotropic wet chemical etching with a mixture of nitric acid $\left(\mathrm{HNO}_{3}\right)$ and hydrofluoric $(\mathrm{HF})$ acid. After the transfer into the $\mathrm{MBE}$, the native oxide is thermally removed by heating to $760{ }^{\circ} \mathrm{C}$ for several minutes. The crystal growth is then initialized with a $85-\mathrm{nm}$ Si buffer layer grown at 520-560 ${ }^{\circ} \mathrm{C}$. Following this, six monolayers (ML) of Ge and a 135-nm-thick Si capping layer are deposited at temperatures of $430{ }^{\circ} \mathrm{C}$ and $410{ }^{\circ} \mathrm{C}$, respectively. For structural investigations, an uncapped layer of islands was grown on the sample surface with the same growth conditions used for the capped island layer. Figure 1(b) shows an atomic force microscope (AFM) image of the surface islands, revealing a bimodal island distribution with smaller "pyramids"17 and larger "domes."17 The emission from the two types of nanostructures is spectrally distinct $^{18}$ and, for the $\mathrm{PhC}$ cavities fabricated in this work, the cavity modes are tuned into resonance with the dome emission at $0.92 \mathrm{eV}$. Thus, we focus on the interaction between the Ge dome islands, which we refer to in the following as " $\mathrm{Ge}$ islands," and the $\mathrm{PhC}$ nanocavity modes.

After growth, $\mathrm{PhC}$ nanostructures were realized using electron beam lithography and subsequent $\mathrm{SF}_{6} / \mathrm{C}_{4} \mathrm{~F}_{8}$ reactive ion etching (RIE) to define hexagonal lattices of air holes with three different periods of $a_{1}=330 \mathrm{~nm}, a_{2}=360 \mathrm{~nm}$, and $a_{3}=390 \mathrm{~nm}$. The scanning electron microscope (SEM) image in Fig. 1(c) shows a typical $\mathrm{PhC}$ with a lattice constant of $a_{3}=390 \mathrm{~nm}$ containing a L3 PhC nanocavity, where the outer holes have been shifted by 0.15 lattice periods. ${ }^{19}$ As a final processing step, the underlying $\mathrm{SiO}_{2}$ is selectively removed by $\mathrm{HF}$ acid to form a freestanding slab membrane.

Optical measurements were performed using a microphotoluminescence $(\mu \mathrm{PL})$ spectroscopy setup. The sample was placed in a liquid-helium flow cryostat for low-temperature investigations. To excite the sample, we used a continuouswave (cw) diode-pumped solid-state laser emitting at $\lambda_{\text {Laser }}=$ $532 \mathrm{~nm}$, which is focused by a $100 \times$ microscope objective $(\mathrm{NA}=0.5)$ to a spot size with a diameter of $\approx 0.8 \mu \mathrm{m}$. The resulting PL signal is collected through the same objective and dispersed by a 32-cm imaging monochromator equipped with a 600 lines/mm grating and a liquid-nitrogen-cooled InGaAs linear diode array.

In Fig. 1(d), we present a typical $\mu$ PL spectrum of the Ge islands emitting in the unpatterned region of the sample. The data, obtained at $T=25 \mathrm{~K}$, can be fitted well by a Gaussian peak with a full width at half maximum (FWHM) of $\approx 50 \mathrm{meV}$ as indicated by the grey line. Due to carrier diffusion, our excitation spot size of $\approx 0.8 \mu \mathrm{m}$ leads to a region with a FWHM of $\approx 1.5 \mu \mathrm{m}$, that generates PL signal, as obtained by performing $\mu \mathrm{PL}$ with spatially separated excitation and detection spots. By comparing this finding with AFM measurements performed on uncapped surface islands, as shown in Fig. $1(\mathrm{~b})$, we estimate that $\approx 100 \mathrm{Ge}$ islands are optically excited by our laser. Hence, the observed spectrum represents the sum of the individual spectra of $N \approx 100$ single Ge islands.

In the following, we will compare the emission intensity of cavity modes with various $Q$ factors to study the cavityemitter coupling. For all experiments reported in this paper, the nominal emission intensity was recorded using an optical excitation power density of either 100 or $600 \mathrm{~kW} / \mathrm{cm}^{2}$ in order to allow a comparative study. Furthermore, it is required that the modes couple spectrally to the same type of islands and, thus, are in a specific spectral window. To do this, we tuned the lattice constant of our $\mathrm{PhC}$ structures during the fabrication process to coarsely adjust the cavity modes and varied the air-hole diameter to fine tune the energy. In Fig. 2(a), we plot $\mu \mathrm{PL}$ spectra recorded at $T=25 \mathrm{~K}$ from a series of L3 $\mathrm{PhC}$ nanocavities with a lattice period of $a_{2}=360 \mathrm{~nm}$ and different air-hole radii, increasing from bottom to top. Six distinct emission lines from the L3 cavity can be observed, five of which are clearly observable in the figure. M1 is the fundamental cavity mode, and M2-M5 are the higher energy modes. ${ }^{20}$ When increasing the air-hole diameter, the modes shift systematically over a range of $\approx 50 \mathrm{meV}$. In general, we did not observe a significant change of the $Q$ factor over the tuning range. For our analysis, we will only consider mode 


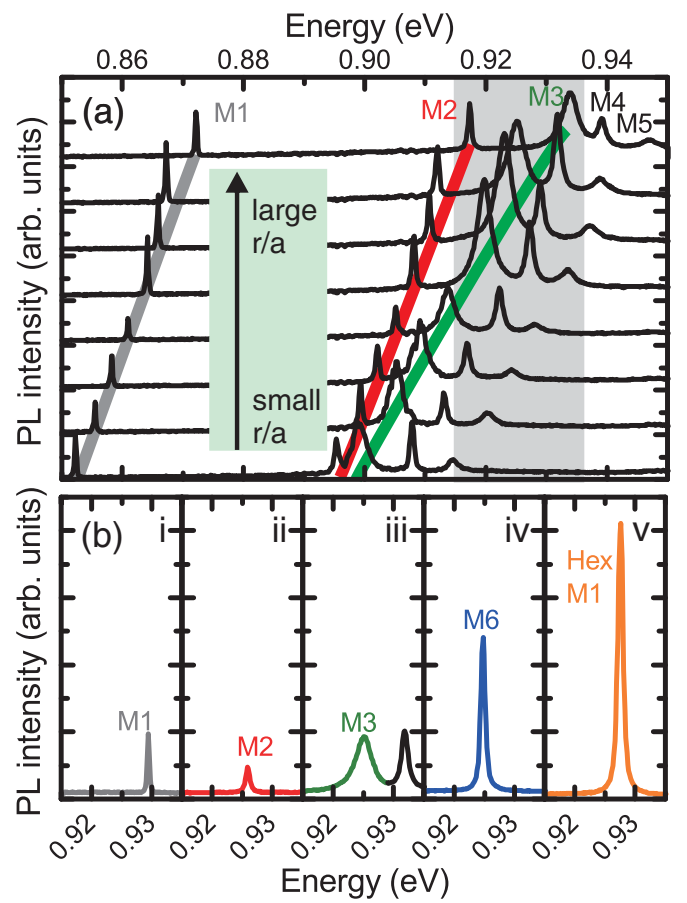

FIG. 2. (Color online) (a) $\mu \mathrm{PL}$ spectra recorded at $T=25 \mathrm{~K}$ from a series of $\mathrm{L} 3 \mathrm{PhC}$ cavities with different air-hole radii, increasing from bottom to top. (b) Mode emission in the spectral range from 0.915 to $0.935 \mathrm{eV}$ [marked grey in (a)] for different cavity modes: (i) fundamental L3 cavity mode (M1), (ii) first higher energy L3 mode (M2), (iii) second higher energy L3 mode (M3), (iv) fifth higher energy L3 mode (M6), and (v) fundamental dipole mode of a hexapole cavity (Hex M1).

emission recorded from samples in the spectral region between 0.915 and $0.935 \mathrm{eV}$, highlighted by the shaded region in Fig. 2(a). As can be seen in the figure, we tune the emission of M2 to M5 into this spectral reference region via the fine tuning method alluded to above. For spectral coarse tuning of the mode emission, we use the PhCs with lattice constants of $a_{1}=330 \mathrm{~nm}$ and $a_{3}=390 \mathrm{~nm}$ to bring M1 and M6 into the spectral region of interest, respectively.

In order to quantitatively compare the intensity of the PL emission, we have to take the mode volume of the nanocavity modes into account. This quantity influences the coupling strength between emitter and cavity and, thus, the cavity-mode $\mu \mathrm{PL}$ intensity. In order to exclude this effect, we consider only cavity modes with similar values of the mode volume in our analysis. Therefore, we performed numerical finite-difference time-domain (FDTD) simulations, ${ }^{21}$ which show that M1, M2, M3, and M6 have comparable mode volumes of $V_{\text {mode }}=0.65 \pm 0.09(\lambda / n)^{3}$, while M4 and M5 exhibit significantly lower mode volumes of $0.30(\lambda / n)^{3}$ and $0.39(\lambda / n)^{3}$, respectively. Therefore, we excluded M4 and M5 from our analysis and consider only M1, M2, M3, and M6. In addition to the L3 cavities, we also fabricated hexapole cavities $^{22}$ emitting in the spectral reference region and showing a mode volume of $V_{\text {mode }}=0.63(\lambda / n)^{3}$ for the fundamental dipole mode (M1 hex) of this structure. Hence, we include this mode in the evaluation presented below. In Fig. 2(b), we plot representative mode emission spectra in the spectral region of
TABLE I. Overview of the simulated properties of the $\mathrm{PhC}$ cavity modes included in our mode intensity versus $Q$ analysis.

\begin{tabular}{lccc}
\hline \hline Mode & $a(\mathrm{~nm})$ & $V_{\text {mode }}(\lambda / n)^{3}$ & $\eta_{\text {coll }}$ \\
\hline M1 & 330 & 0.74 & 0.197 \\
M2 & 360 & 0.73 & 0.108 \\
M3 & 360 & 0.57 & 0.437 \\
M6 & 390 & 0.63 & 0.468 \\
M1 hex & 330 & 0.63 & 0.466 \\
\hline \hline
\end{tabular}

interest from 0.915 to $0.935 \mathrm{eV}$ for a number of different cavity modes: (i) the fundamental L3 cavity mode (M1), (ii) the first higher energy L3 mode (M2), (iii) the second higher energy L3 mode (M3), (iv) the fifth higher energy L3 mode (M6), and (v) the fundamental dipole mode of a hexapole cavity (Hex M1). The PL intensity scale is the same for all graphs.

In addition, we need to take the far-field emission profile of the different cavity modes into account since the far-field profile influences the collection efficiency $\eta_{\text {coll }}$ of our optical detection system. As a result, the fraction of light emitted to one hemisphere that is collected by our microscope objective differs for different cavity-mode profiles. We obtained the values of $\eta_{\text {coll }}$ for the different cavity modes investigated using FDTD simulations. Table I summarizes the simulation results for the cavity modes that we include in our analysis. Here, $a$ is the lattice constant used for coarse shifting the mode emission, $V_{\text {mode }}$ the mode volume, and $\eta_{\text {coll }}$ the photon collection efficiency.

Finally, we need to exclude measurement errors such as imprecise positioning of the excitation spot on the cavity or varying fabrication quality of different $\mathrm{PhCs}$, all of which would influence the intensity of the PL signal. Hence, we measured three to seven different photonic crystal nanocavities for each of the mode types emitting in the spectral window from 0.915 to $0.935 \mathrm{eV} .{ }^{23}$ The mode intensity is extracted by fitting a Lorentzian peak to the spectral profile and averaging the peak areas for modes of the same type. The background stemming from the uncoupled Ge-island emission or from spectrally closely spaced modes (M3 and M4) is subtracted to separate the cavity emission from the background.

In order to obtain the emitted mode intensity $I_{\text {mode }}$, we correct the measured intensity $I_{\text {measured }}$ for the mode volume $\left(V_{\text {mode }}\right)$ related change of the number of islands coupled to the cavity, for the angular collection efficiency $\left(\eta_{\text {coll }}\right)$ and for the mode degeneracy $D$ (Ref. 24):

$$
I_{\text {mode }}=\frac{I_{\text {measured }}}{V_{\text {mode }} \eta_{\text {coll }} D} .
$$

In Fig. 3, we plot the obtained average emitted mode intensities $\left(I_{\text {mode }}\right)$ as a function of inverse $Q$ and cavity-mode linewidth $\gamma_{a}\left(Q=\omega_{\text {cav }} / \gamma_{a}\right)$ for excitation power densities of $100 \mathrm{~kW} / \mathrm{cm}^{2}$ (open triangles, dotted line as guide for the eye) and $600 \mathrm{~kW} / \mathrm{cm}^{2}$ (filled circles, solid line as guide for the eye), normalized to the maximum value for each set of data points. The error bars account for the standard deviation of the intensity and $Q$ factor distribution since each point is the average of several independent measurements performed on a number of cavities. The (red) shaded area marks the region that can not be accessed by our experimental setup due to 


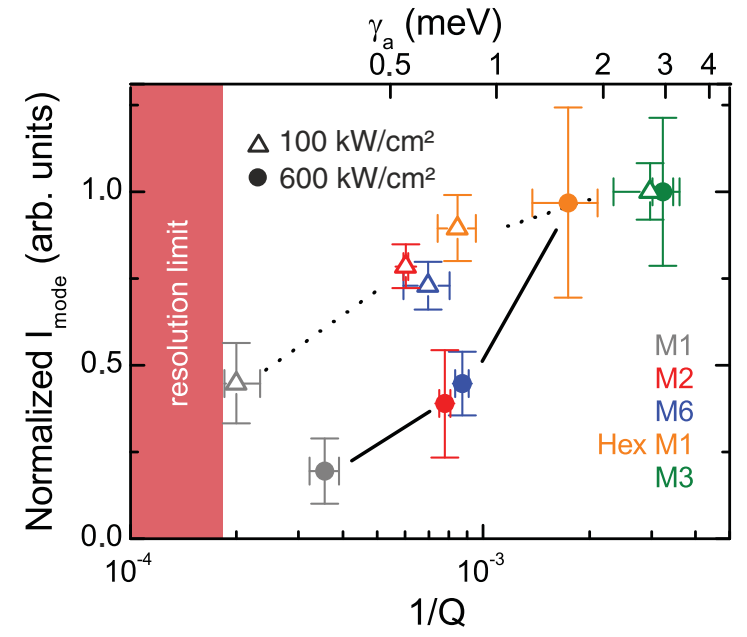

FIG. 3. (Color online) Average mode emission intensity as a function of inverse cavity $Q$ factor (cavity linewidth $\gamma_{a}$ ) for excitation power densities of $100 \mathrm{~kW} / \mathrm{cm}^{2}$ (open triangles, dotted line as guide for the eye) and $600 \mathrm{~kW} / \mathrm{cm}^{2}$ (filled circles, solid line as guide for the eye), normalized to the maximum value for each set of data points. Each point is obtained by averaging over several modes emitting in the spectral range between 0.915 and $0.935 \mathrm{eV}$. The error bars display the standard deviation. The (red) shaded area marks the region that cannot be accessed by our experimental setup due to the limited spectral resolution.

the limited spectral resolution of $\approx 0.17 \mathrm{meV}$. An excitation power density of $100 \mathrm{~kW} / \mathrm{cm}^{2}$ is the minimum pumping power at which we receive sufficient signal to obtain reliable statistics for all cavity modes. At this power, when decreasing the linewidth from $3 \mathrm{meV}(Q \approx 300)$ to $1 \mathrm{meV}(Q \approx 900)$, we observe a slight decrease of $25 \%$ in emission intensities. The high- $Q$ mode M1 shows a $45 \%$ decrease of intensity. However, the limited spectral resolution of our setup prohibits measurement of the actual linewidth. The linewidth of M1 might be considerably smaller than our detection limit. At higher excitation power densities of $600 \mathrm{~kW} / \mathrm{cm}^{2}$, we clearly observe a more pronounced trend of decreasing intensities with decreasing cavity linewidth (increasing $Q$ ). The average emission intensity of cavities with $\gamma_{a}>1.5 \mathrm{meV}(Q<600)$ are similar. In contrast, for $\gamma_{a}<1.5 \mathrm{meV}(Q>600)$, we observe a progressive and systematic decrease in $I_{\text {mode }}$ with decreasing cavity linewidth (increasing $Q$ factors). For the fundamental mode of the L3 cavity (M1), which is the mode with the smallest linewidth, $I_{\text {mode }}$ is strongly reduced to approximately $1 / 5$ of the intensity of Hex M1 and M3. In order to understand this very clear experimental observation, we introduce in the following section a cavity quantum electrodynamics (QED) model based on a dissipative master equation.

\section{THEORY}

In this section, we present a cavity QED model that describes our system of an ensemble of Ge islands coupled to a cavity mode. Ge islands are expected to have a very weak transition dipole moment due to the spatial separation between electrons captured at the $\mathrm{Si}-\mathrm{Ge}$ interface and the holes localized in the Ge islands (type-II band alignment). ${ }^{25}$
Hence, the coupling parameter $g$ that describes the interaction between emitter and cavity is expected to be very small. In the following, the parameters will be denoted as illustrated schematically in Fig. 4(a), where $N$ is the number of Ge islands coupled to the cavity mode, $\gamma_{b}$ is the FWHM of the emission of a single Ge island, $\sigma$ is the FWHM of the spectral island ensemble distribution, $\gamma_{a}$ is the FWHM of the cavity emission, and $\tilde{\Delta}$ is the effective spectral detuning between the center of the cavity and the center of the island ensemble. To capture the essential physics that determines the dynamics of such a system, i.e., $N$ Ge islands weakly coupled to a cavity mode, we turn to the simplest possible picture provided by a dissipative master equation (in units of $\hbar=1$ ) $i \partial_{t} \rho=\mathcal{L} \rho$ for the density matrix $\rho$ with $\mathcal{L} \rho=[H, \rho]+\mathcal{L}_{\gamma} \rho+\mathcal{L}_{P} \rho$, where $H$ is the Hamiltonian that describes the dynamics of the islands' nanocavity system:

$$
H=H_{\text {free }}+\sum_{i=1}^{N} g_{i}^{a}\left(b_{i}^{\dagger} a+b_{i} a^{\dagger}\right)+\sum_{i=1}^{N} V_{i} b_{i}^{\dagger} b_{i}^{\dagger} b_{i} b_{i},
$$

with $H_{\text {free }}=\omega_{a} a^{\dagger} a+\sum_{i=1}^{N} \omega_{i} b_{i}^{\dagger} b_{i}$ being the free dynamics of the modes and

$$
\mathcal{L}_{\gamma_{c}} \rho=\frac{\gamma_{c}}{2}\left(2 c \rho c^{\dagger}-c^{\dagger} c \rho-\rho c^{\dagger} c\right)
$$
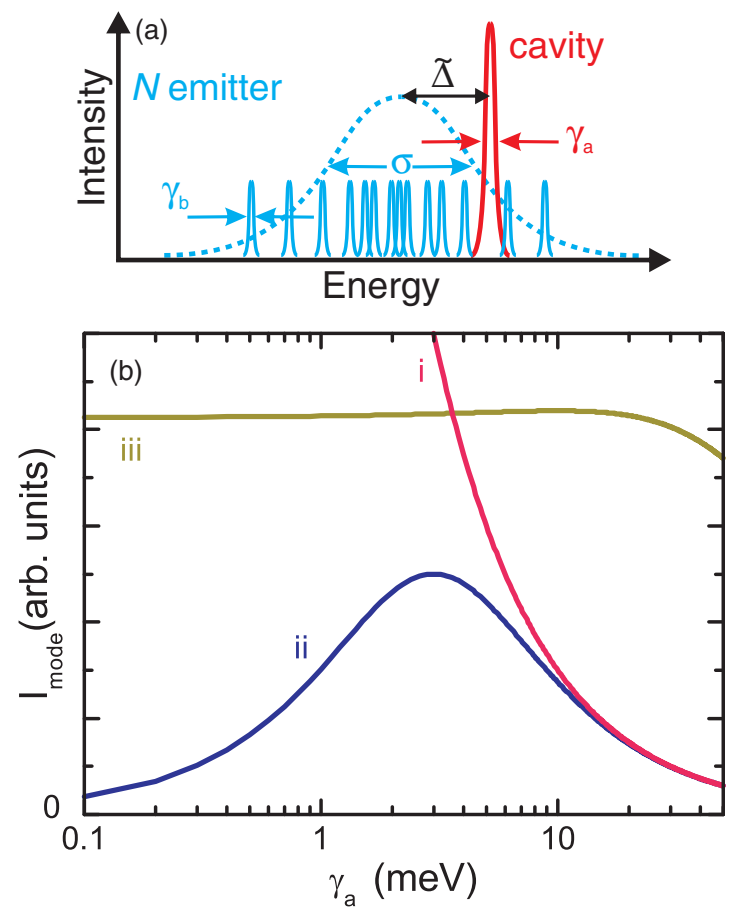

FIG. 4. (Color online) (a) Schematic illustration of the parameters used for the theoretical model of $N$ emitters coupled to a cavity: cavity linewidth $\gamma_{a}$, emitter linewidth $\gamma_{b}$, emitter distribution $\sigma$, and effective ensemble cavity detuning $\tilde{\Delta}$. (b) Theoretical trends in various limiting cases of the cavity QED light-matter coupling: (i) cavity at resonance ( $\tilde{\Delta}=0)$ and (ii) at finite detuning $(\tilde{\Delta}=1.5 \mathrm{meV})$ to a spectrally narrow emitter ensemble with a FWHM of $\Gamma_{b}+\sigma=0.01 \mathrm{meV}$. (iii) Cavity at large detuning $(\tilde{\Delta}=30 \mathrm{meV})$ to a broad emitter ensemble with an effective ensemble linewidth of $\Gamma_{b}+\sigma=50 \mathrm{meV}$, which corresponds to our system. 
is the Lindblad operator for the cavity modes, where $c=a$, $b_{i}$ is the decay of the modes. Pumping is included in the same way, as an incoherent source of excitation with Lindblad term $\mathcal{L}_{P_{i}} \rho=\frac{P_{i}}{2}\left(2 b_{i}^{\dagger} \rho b_{i}-b_{i} b_{i}^{\dagger} \rho-\rho b_{i} b_{i}^{\dagger}\right)$. The nonlinear term $b_{i}^{\dagger} b_{i}^{\dagger} b_{i} b_{i}$ describes phase-space filling by approximating the real energy level structures of the island ${ }^{26}$ to be an an equally spaced ladder of levels ${ }^{27}$ with spacing $V_{i}$ for the $i$ th dot. A mean-field approximation $\left\langle b_{i}^{\dagger} b_{i} a^{\dagger} b_{i}\right\rangle \approx\left\langle b_{i}^{\dagger} b_{i}\right\rangle\left\langle a^{\dagger} b_{i}\right\rangle$ is performed that allows us to truncate the equations of motion self-consistently. The main effect of this term is to provide an effective detuning between the cavity mode and the spectral center of the emitter ensemble distribution, as well as an effective broadening of the emitter line. The steady-state population in the cavity $n_{a}=\lim _{t \rightarrow \infty} \operatorname{Tr}\left[\rho(t) a^{\dagger} a\right]$ is then obtained, from which follows the number of photons emitted per unit time $I_{\text {mode }}=\gamma_{a} n_{a}$. This is the main quantity of interest in our experiment. A closed-form expression can be obtained for a Lorentzian distribution of islands (a Gaussian distribution is nonintegrable) that shows the combined effect of detuning $\Delta=\omega_{a}-\sum_{i=1}^{N} \omega_{i}$ between the cavity mode and the average position of the islands and their distribution $\sigma$ :

$$
I_{\text {mode }}=\frac{P_{b}}{\Gamma_{b}} \frac{\gamma_{a}}{\left(\gamma_{a}+\Gamma_{b}+\sigma\right)} \frac{4 g_{\text {eff }}^{2}}{\gamma_{a}},
$$

where $P_{b}$ is the rate at which the emitters are being pumped. Here, we have retained the leading term only in the coupling strength $g$ since it is very small in our systems, and have defined the effective coupling

$$
g_{\text {eff }}^{2}=\frac{N g^{2}}{1+\left(\frac{\tilde{\Delta}}{\frac{\gamma_{a}+\Gamma_{b}+\sigma}{2}}\right)^{2}},
$$

where $\tilde{\Delta}=\sqrt{\Delta^{2}+\left[\left\langle\sum_{i} b_{i}^{\dagger} b_{i} V_{i}\right\rangle / N\right]^{2}}$ is the effective detuning that includes the interactions, and $\Gamma_{b}$ is the effective broadening of a single emitter that, following Bose statistics, reads as $\gamma_{b}-P_{b}$. These two new parameters are, however, to be considered the natural and independent ones that describe the system rather than the microscopic ones from which they stem. $\Delta$ and $\gamma_{b}$ do not play a direct role anymore.

In terms of these effective parameters, Eq. (4) is closely related to that of a single emitter coupled to a cavity (and reduces to it when $N=1, V_{i}=0$, and $\left.\sigma=0\right) .{ }^{28}$ The first term in Eq. (4), $P_{b} / \Gamma_{b}$, is the effective population that builds up among the islands. The second term $\gamma_{a} /\left(\gamma_{a}+\Gamma_{b}+\sigma\right)$ is the fraction of these excitations that is available to excite the cavity. The third term $4 g_{\text {eff }}^{2} / \gamma_{a}$ dominates the dynamics and governs the intensity-linewidth trend.

In Fig. 4(b), we plot Eq. (4) for various limiting cases of the cavity QED light-matter coupling: (i) cavity at resonance $(\tilde{\Delta}=0)$ and (ii) at finite detuning $(\tilde{\Delta}=1.5 \mathrm{meV})$ to a spectrally narrow emitter ensemble with an effective ensemble linewidth of $\Gamma_{b}+\sigma=0.01 \mathrm{meV}$. In curve (iii), we plot Eq. (4) for a cavity at large detuning $(\tilde{\Delta}=30 \mathrm{meV})$ to a broad emitter ensemble distribution with $\Gamma_{b}+\sigma=50 \mathrm{meV}$. These parameters correspond closely to the expected reality for our system [cf. Fig. 1(d)]. For very weak coupling $(g \rightarrow 0)$, the intensity of the emitted light increases with the quality of the cavity $\left(\gamma_{a} \rightarrow 0\right)$ if the cavity mode is placed at resonance with a spectrally narrow emitter ensemble $(\tilde{\Delta}=0)$. This can clearly be seen by curve (i) in Fig. 4(b). At nonzero detuning with finite spectral mismatch $\tilde{\Delta}$, the effective coupling becomes larger with decreasing $Q$ (increasing $\gamma_{a}$ ) due to an increasing overlap between the detuned cavity mode and the collective set of islands, with effective linewidth $\Gamma_{b}+\sigma$. Hence, making the cavity $Q$ worse gives rise to an increase of the mode emission intensity. For very low $Q$ (large $\gamma_{a}$ ), the intensity decreases since the coupling between the emitter ensemble and the cavity gets weak. This is shown representatively by curve (ii) in Fig. 4(b). The trends shown in curves (i) and (ii) can be observed only when the effective ensemble linewidth $\Gamma_{b}+\sigma$ of the island ensemble is comparable to the linewidth $\gamma_{a}$ of the cavity mode. In strong contrast, our system has an ensemble linewidth of $\approx 50 \mathrm{meV}$, which is more than an order of magnitude larger than the cavity linewidth. For this case, our model predicts that the emitted intensity should exhibit a plateau for the regime of cavity-mode linewidths that we observe in our experiment $\left(0.3 \mathrm{meV}<\gamma_{a}<3 \mathrm{meV}\right)$, quite independently of detuning. This is shown by curve (iii) in Fig. 4(b). Clearly, experimentally we do not observe the theoretically predicted plateau for the high- $Q$ region, but rather a decrease in emission intensity as presented in Fig. $3{ }^{29}$ So far, our cavity QED model takes only photon emission from the islands into the cavity mode into account, while in the real experiment, we have additional effects such as photon reabsorption, annihilating the photon before it escapes the cavity. With increasing cavity $Q$, the time the photon remains inside the cavity increases and, hence, absorption effects are expected to play an increasingly important role for high $Q$ cavities. This is supported by the fact that M1 is expected to have a high $Q$ factor of $Q_{\text {sim }} \approx 70000$ according to our FDTD simulations, but we observe a significantly smaller $Q$ factor in our experiment of $Q_{\exp } \approx 2800$ for an excitation power density of $600 \mathrm{~kW} / \mathrm{cm}^{2}$.

Due to the spatial separation between electron and hole wavefunction and the indirect optical transition in $k$-space, we expect the resonant absorption of the Ge islands to be too weak to explain the $Q$ factor saturation in our experiment. This expectation is supported by the observation that the $Q$ factor of the nanocavity emission is independent of the spectral position relative to the Ge island ensemble. If reabsorption of photons by $\mathrm{Ge}$ islands would play a dominant role, we would expect to observe an increase of $Q$ factors for cavity modes emitting at the low-energy side of the ensemble since the probability of photon reabsorption decreases there. However, free carrier absorption (FCA) can cause photon reabsorption since we generate a large density of charge carriers in the vicinity of the nanocavity, as we optically excite with a high power density of $600 \mathrm{~kW} / \mathrm{cm}^{2}$. Such a high optical pumping intensity is required to observe a strong PL signal. FCA has been reported and investigated for optical cavities of III-V systems ${ }^{31,32}$ as well as for Si-based photonic systems such as Si nanocrystals. ${ }^{33} \mathrm{El}$ Kurdi et al. ${ }^{14}$ have observed mode linewidth broadening with increasing excitation power in Si photonic crystal nanocavities with embedded Ge islands, which they attribute to FCA. However, neither an investigation on the correlation between free carrier absorption and the observed emitted PL intensity nor an attempt to describe the experimentally observed mode 
emission by a cavity QED model have been made for such a system.

To describe our experiment, we take reabsorption of photons into account as a competing process between the escape of a photon out of a cavity with a photon escape time $\tau_{\text {esc }}$, given by the intrinsic $Q$ factor of the cavity, and the photon reabsorption time $\tau_{\mathrm{abs}}$. The total photon loss time $\tau_{\mathrm{a}}$, given by $1 / \tau_{\mathrm{a}}=$ $1 / \tau_{\text {esc }}+1 / \tau_{\text {abs }}$, determines the experimentally observed $Q$ factor and, therefore, the cavity linewidth $\gamma_{a}$ measured in PL. Hence, the emitted mode intensity $I_{\text {mode }}^{\text {abs }}$ in the presence of photon reabsorption can be expressed as

$$
\begin{aligned}
I_{\text {mode }}^{\mathrm{abs}}\left(\gamma_{a}\right) & =I_{\text {mode }}\left(\gamma_{\mathrm{esc}}\right) \frac{1 / \tau_{\mathrm{esc}}}{1 / \tau_{\mathrm{esc}}+1 / \tau_{\mathrm{abs}}} \\
& =I_{\text {mode }}\left(\gamma_{a}-\gamma_{\mathrm{abs}}\right)\left(1-\frac{\gamma_{\mathrm{abs}}}{\gamma_{a}}\right)
\end{aligned}
$$

$I_{\text {mode }}^{\text {abs }}\left(\gamma_{a}\right)$ denotes the emitted PL intensity of a cavity with a linewidth of $\gamma_{a}$ when including absorption, $I_{\text {mode }}\left(\gamma_{\text {esc }}\right)$ denotes the emitted PL intensity of the same cavity without absorption and, hence, a smaller cavity linewidth $\gamma_{\mathrm{esc}}$, which is solely determined by $1 / \tau_{\text {esc }}$. The last term describes the competing process between photon escape from the cavity and photon reabsorption, where $\gamma_{\mathrm{abs}}$ is the cavity linewidth given by the absorption.

\section{DISCUSSION}

In Fig. 5, we present the theoretically predicted trend of the mode emission intensity as a function of cavity linewidth $\gamma_{a}$ for our system in the absence of photon reabsorption [dotted line, cf. Fig. 4(b)]. Furthermore, we plot a fit (full line) of the model to the experimental data points (gray, points) obtained at an excitation power density of $600 \mathrm{~kW} / \mathrm{cm}^{2}$ from Fig. 3 , using the same parameters and now including reabsorption of photons as described by Eq. (6). The dataset obtained

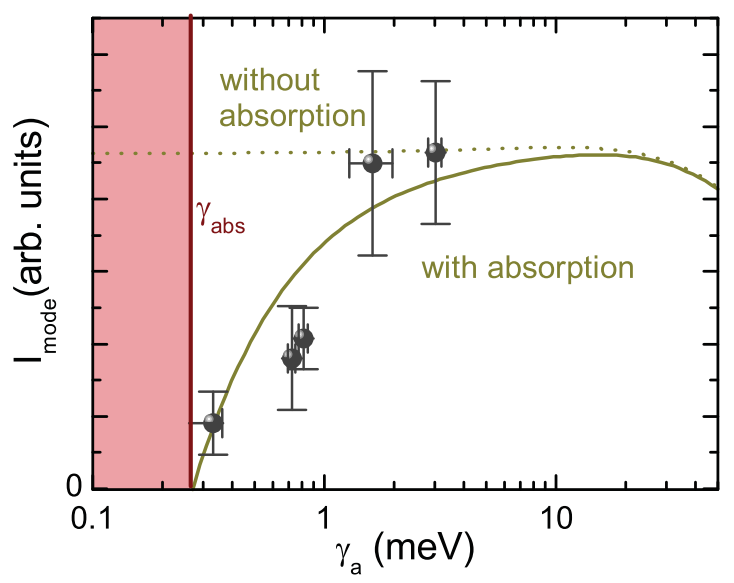

FIG. 5. (Color online) Mode emission intensity as a function of cavity linewidth $\gamma_{a}$ : (dotted line) Theoretically predicted trend for our system with an emitter ensemble distribution with $\Gamma_{b}+\sigma=50 \mathrm{meV}$ and an ensemble cavity detuning of $\tilde{\Delta}=30 \mathrm{meV}$, in the absence of photon reabsorption and (solid line) including reabsorption of photons with an absorption of $\gamma_{\mathrm{abs}}=0.27 \pm 0.05 \mathrm{meV}$ together with the experimental data points obtained at an excitation power density of $600 \mathrm{~kW} / \mathrm{cm}^{2}$ from Fig. 3 . at $100 \mathrm{~kW} / \mathrm{cm}^{2}$ is not suitable for a fitting procedure since we can not determine the linewidth of M1 due to limited spectral resolution and the trend of the remaining data points is too weak. We use $\gamma_{\mathrm{abs}}$ and the normalization of $I_{\text {mode }}$ as fitting parameters. The theoretically predicted trend follows our experimental data by showing a decrease of emission intensity with decreasing $\gamma_{a}$ (increasing $Q$ factors). Obviously, we do not expect to observe mode emission for linewidths $\gamma_{a}$ smaller than $\gamma_{\mathrm{abs}}$, as highlighted by the (red) shaded area. From the fit, we extract $\gamma_{\mathrm{abs}}=0.27 \pm 0.05 \mathrm{meV}$, which corresponds to a photon reabsorption time of $\tau_{\mathrm{abs}}=2.4 \pm 0.6 \mathrm{ps}$. The presence of FCA is supported by the fact that we observe, with increasing optical pumping power, a broadening of the cavity modes. This is an effect that El Kurdi et al. ${ }^{14}$ have observed at similar excitation power densities for Si photonic crystal nanocavities with embedded Ge islands. An example of such pump-power dependent investigations for M2 is shown in Fig. 6(a), where we plot the mode linewidth of M2, $\gamma_{a}^{\mathrm{M} 2}$, as a function of optical excitation power density. In our system, at low optical excitation power densities, the linewidth of M2 stays constant at $\gamma_{\mathrm{esc}}^{\mathrm{M} 2}=0.74 \pm 0.02 \mathrm{meV}$ (marked by the horizontal dotted line), as it is solely determined by the intrinsic photon escape time $\tau_{\text {esc }}$ of the cavity mode. When increasing the excitation power density above $250 \mathrm{~kW} / \mathrm{cm}^{2}$, we observe a strong increase in linewidth. At $600 \mathrm{~kW} / \mathrm{cm}^{2}$ (marked by the green vertical line), which corresponds to the optical excitation power density we used in our comparative experiment, $\gamma_{a}^{\mathrm{M} 2}$ is increased to $0.97 \pm 0.02 \mathrm{meV}$. When further increasing the

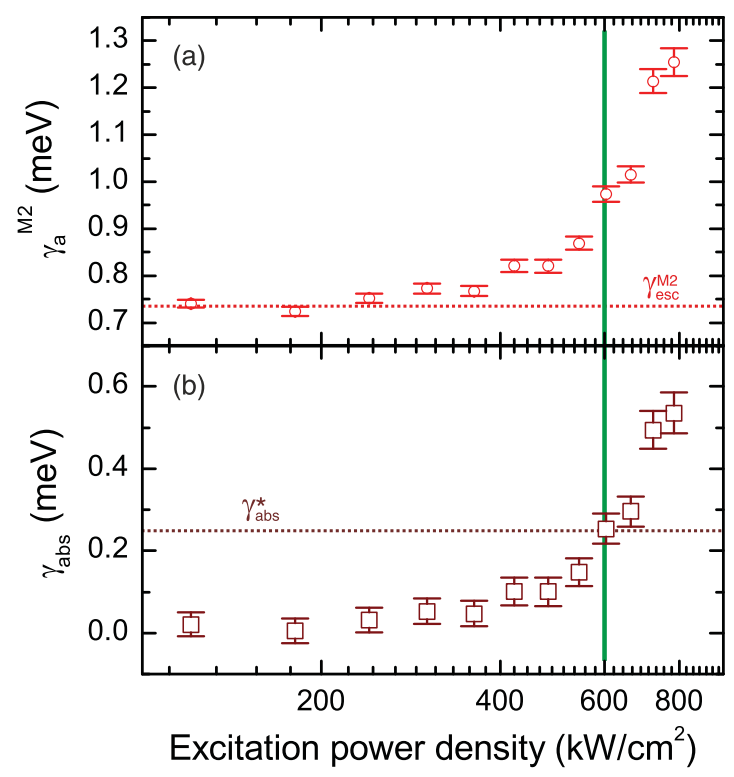

FIG. 6. (Color online) (a) Cavity-mode linewidth of M2, $\gamma_{a}^{\mathrm{M} 2}$, as a function of optical excitation power density. The vertical line marks the optical excitation power density that we used in our comparative study $\left(600 \mathrm{~kW} / \mathrm{cm}^{2}\right)$. The horizontal dotted line marks the intrinsic linewidth $\gamma_{\mathrm{esc}}^{\mathrm{M} 2}$ of M2. (b) $\gamma_{\mathrm{abs}}$, extracted from $\gamma_{a}^{\mathrm{M} 2}$, as a function of optical excitation power density. The vertical line marks the optical excitation power density we used in our comparative study $\left(600 \mathrm{~kW} / \mathrm{cm}^{2}\right)$. The horizontal dotted line marks the extracted absorption linewidth of $\gamma_{\mathrm{abs}}^{\star}=0.23 \pm 0.04 \mathrm{meV}$ at this power. 
optical excitation power, the absorption coefficient is increased due to heating caused by the excitation laser. This leads to a strong increase of optically generated charge carriers inside the cavity and, as a result, $\gamma_{a}^{\mathrm{M} 2}$ increases strongly. Since we can extract the intrinsic linewidth of M2 from the measurements at low power, we can extract the reabsorption $\gamma_{\mathrm{abs}}$, using $\gamma_{\mathrm{abs}}=$ $\gamma_{a}^{\mathrm{M} 2}-\gamma_{\mathrm{esc}}^{\mathrm{M} 2}$. In Fig. 6(b), we plot $\gamma_{\mathrm{abs}}$ as a function of optical excitation power density. At $600 \mathrm{~kW} / \mathrm{cm}^{2}$ (marked by the green vertical line), which corresponds to the optical excitation power density we used in our comparative experiment, we obtain $\gamma_{\mathrm{abs}}^{\star}=0.23 \pm 0.04 \mathrm{meV}$, corresponding to a photon reabsorption time of $\tau_{\mathrm{abs}}^{\star}=2.9 \pm 0.6 \mathrm{ps}$. This is in remarkable agreement with $\tau_{\text {abs }}=2.4 \pm 0.6 \mathrm{ps}$, as extracted from the fit in Fig. 5. Thus, these observations lend strong support to the argument that FCA limits the emission enhancement. A major conclusion of our paper is that the overall emitted intensity from optically pumped $\mathrm{PhC}$ nanocavities with embedded $\mathrm{Ge}$ islands is mainly limited by FCA, caused by the high charge carrier density from strong optical excitation. Concerning the external quantum efficiency, high $Q$ cavities are actually performing worse than low $Q$ cavities due to the increased probability of reabsorption of photons emitted into the cavity.

\section{CONCLUSION}

We reported a comparative study of PL emission from $\mathrm{PhC}$ nanocavities with embedded Ge islands by low-temperature $\mu \mathrm{PL}$ spectroscopy. First, we investigated a number of different L3 and hexapole PhC cavity modes with various $Q$ factors. For a valid comparison, we considered only mode emission in a well-defined spectral region, corrected for the mode volume related change of emitters pumping the cavity mode and the far-field radiation pattern. Under conditions of strong optical pumping, with increasing $Q$ factors we observed a decrease of the PL intensity emitted by the cavity mode. This trend is less pronounced for lower excitation power densities. We then introduced a cavity QED model based on a dissipative master equation to understand the dynamics of an ensemble of emitters, which are in highly excited states due to strong optical pumping and in very weak coupling with the cavity mode. With this model, we could identify various regimes of PL intensity versus $Q$ factor trends, depending on the emitter ensemble distribution and the spectral emitter-cavity detuning. By comparing the theoretically predicted trend of our system with the experimental data, we concluded that reabsorption of photons emitted into the cavity limits the emission enhancement via the cavity mode. We extracted from the model a photon reabsorption time of $\tau_{\mathrm{abs}}=2.4 \pm 0.6 \mathrm{ps}$ for an excitation power density of $600 \mathrm{~kW} / \mathrm{cm}^{2}$ and concluded, by comparing this value to the photon reabsorption time $\tau_{\mathrm{abs}}^{\star}=$ $2.9 \pm 0.6$ ps extracted from power-dependent measurements, that the absorption is caused by free carriers. Therefore, cavity modes with high $Q$ factors and, hence, long photon lifetimes exhibit a reduced radiative quantum efficiency compared to cavity modes with low $Q$ factors.

\section{ACKNOWLEDGMENTS}

We would like to thank E. del Valle (University of Southampton, Southampton, United Kingdom) and P. Senellart (Laboratoire de Photonique et Nanostructures, Marcoussis, France) for fruitful discussions. We acknowledge financial support from the German Excellence Initiative via the Nanosystems Initiative Munich (NIM), IEF project SQOD, and the TUM International Graduate School of Science and Engineering (IGSSE). *hauke@wsi.tum.de

${ }^{1}$ B. Jalali, Nat. Photonics 1, 193 (2007).

${ }^{2}$ B. Jalali and S. Fathpour, J. Lightwave Technol. 24, 4600 (2006).

${ }^{3}$ R. Soref, IEEE J. Sel. Top. Quantum Electron. 12, 1678 (2006).

${ }^{4}$ N. Izhaky, M. T. Morse, S. Koehl, O. Cohen, D. Rubin, A. Barkai,

G. Sarid, R. Cohen, and M. J. Paniccia, IEEE J. Sel. Top. Quantum Electron. 12, 1688 (2006).

${ }^{5}$ L. Kimerling, Appl. Surf. Sci. 159, 8 (2000).

${ }^{6}$ L. Pavesi, Silicon Photonics (Springer, Berlin, 2004).

${ }^{7}$ S. Iwamoto, Y. Arakawa, and A. Gomyo, Appl. Phys. Lett. 91, 211104 (2007).

${ }^{8}$ M. Fujita, Y. Tanaka, and S. Noda, IEEE J. Sel. Top. Quantum Electron. 14, 1090 (2008).

${ }^{9}$ N. Hauke, T. Zabel, K. Müller, M. Kaniber, A. Laucht, D. Bougeard, G. Abstreiter, J. J. Finley, and Y. Arakawa, New J. Phys. 12, 053005 (2010).

${ }^{10}$ R. L. Savio, S. L. Portalupi, D. Gerace, A. Shakoor, T. F. Krauss, L. O'Faolain, L. C. Andreani, and M. Galli, Appl. Phys. Lett. 98, 201106 (2011).

${ }^{11}$ X. Li, P. Boucaud, X. Checoury, O. Kermarrec, Y. Campidelli, and D. Bensahel, J. Appl. Phys. 99, 023103 (2006).
${ }^{12}$ J. S. Xia, Y. Ikegami, Y. Shiraki, N. Usami, and Y. Nakata, Appl. Phys. Lett. 89, 201102 (2006).

${ }^{13}$ P. Boucaud, M. El Kurdi, S. David, X. Checoury, X. Li, T. P. Ngo, S. Sauvage, D. Bouchier, G. Fishman, O. Kermarrec, Y. Campidelli, D. Bensahel, T. Akatsu, C. Richtarch, and B. Ghyselen, Thin Solid Films 517, 121 (2008).

${ }^{14}$ M. El Kurdi, X. Checoury, S. David, T. P. Ngo, N. Zerounian, P. Boucaud, O. Kermarrec, Y. Campidelli, and D. Bensahel, Opt. Express 16, 8780 (2008).

${ }^{15}$ J. Xia, R. Tominaga, S. Fukamitsu, N. Usami, and Y. Shiraki, Jpn. J. Appl. Phys. 48, 022102 (2009).

${ }^{16}$ [www.soitec.com].

${ }^{17}$ F. Ross, Science 287, 594 (2000).

${ }^{18}$ R. Magalhães-Paniago, G. Medeiros-Ribeiro, A. Malachias, S. Kycia, T. I. Kamins, and R. S. Williams, Phys. Rev. B 66, 245312 (2002).

${ }^{19}$ Y. Akahane, T. Asano, B. S. Song, and S. Noda, Nature (London) 425, 944 (2003).

${ }^{20}$ A. R. A. Chalcraft, S. Lam, D. O’Brien, T. F. Krauss, M. Sahin, D. Szymanski, D. Sanvitto, R. Oulton, M. S. Skolnick, A. M. Fox, D. M. Whittaker, H.-Y. Liu, and M. Hopkinson, Appl. Phys. Lett. 90, 241117 (2007). 
${ }^{21}$ [www.rsoftdesign.com].

${ }^{22}$ H. Ryu, M. Notomi, and Y. Lee, Appl. Phys. Lett. 83, 4294 (2003).

${ }^{23}$ In our experiment, we apply very strict requirements on the choice of cavity modes that are finally included in our analysis. Most importantly, we consider only mode emission in a spectrally narrow window for a comparative analysis. It is very difficult to deterministically fabricate cavity with a precise emission wavelength. Therefore, we fabricated 240 photonic crystals and characterized the mode emission for each one. Out of these 240 photonic crystals, we filtered the results and chose only cavities whose mode emission occurred in narrow spectral window from 0.915 to $0.935 \mathrm{eV}$ for our analysis. This is essential since different cavities can only be compared with one another if they couple to the same subset of the Ge-island emission spectrum. This resulted in only three to seven different modes that can fulfill this requirement for each cavity.

${ }^{24}$ The dipole mode in a hexapole cavity is twofold degenerate.

${ }^{25}$ M. Larsson, A. Elfving, W.-X. Ni, G. V. Hansson, and P. O. Holtz, Phys. Rev. B 73, 195319 (2006).
${ }^{26}$ P. Boucaud, S. Sauvage, M. Elkurdi, E. Mercier, T. Brunhes, V. LeThanh, D. Bouchier, O. Kermarrec, Y. Campidelli, and D. Bensahel, Phys. Rev. B 64, 155310 (2001).

${ }^{27}$ E. del Valle, F. P. Laussy, F. M. Souza, and I. A. Shelykh, Phys. Rev. B 78, 085304 (2008).

${ }^{28}$ F. P. Laussy, E. del Valle, and C. Tejedor, Phys. Rev. B 79, 235325 (2009).

${ }^{29}$ A system in the "bad emitter regime" ${ }^{30}$ would show a decrease of mode intensity with increasing $Q$ since the cavity acts as a spectral filter. However, assuming our system to be in the bad emitter limit leads to unrealistically high values of $g$ (in the order of meV). Hence, we exclude that the intensity decrease we observe is caused by such spectral filter effects.

${ }^{30}$ A. Pitanti, M. Ghulinyan, D. Navarro-Urrios, G. Pucker, and L. Pavesi, Phys. Rev. Lett. 104, 103901 (2010).

${ }^{31}$ E. Schubert, N. Hunt, R. Malik, M. Micovic, and D. Miller, J. Lightwave Technol. 14, 1721 (1996).

${ }^{32}$ G. Scarpa, N. Ulbrich, A. Sigl, M. Bichler, D. Schuh, M. Amann, and G. Abstreiter, Phys. E (Amsterdam) 13, 844 (2002).

${ }^{33}$ R. D. Kekatpure and M. L. Brongersma, Nano Lett. 8, 3787 (2008). 